\title{
A phase 1 dose-escalating study of pegylated recombinant human arginase 1 (Peg-rhArg1) in patients with advanced hepatocellular carcinoma
}

\author{
Thomas Yau • P. N. Cheng • Pierre Chan • \\ William Chan • Li Chen • Jimmy Yuen • Roberta Pang •
}

S. T. Fan • Ronnie T. Poon

Received: 5 January 2012 / Accepted: 22 February 2012 / Published online: 17 March 2012

(C) The Author(s) 2012. This article is published with open access at Springerlink.com

Summary Background Hepatocellular carcinoma (HCC) cells are auxotrophic for arginine, depletion of which leads to tumour regression. The current study evaluated safety, pharmacokinetics $(\mathrm{PK}) /$ pharmacodynamics $(\mathrm{PD})$ parameters, and potential anti-tumor activity of pegylated recombinant human arginase 1 (peg-rhArg1) in advanced HCC patients. Methods Eligibility criteria included advanced HCC with measurable lesions, Child-Pugh A or B, and adequate organ function. Initial single IV bolus was followed by weekly doses of peg-rhArgI escalated from $500 \mathrm{U} / \mathrm{kg}$ to $2500 \mathrm{U} / \mathrm{kg}$ in a $3+3$ design. Results Fifteen patients were enrolled at weekly doses of $500 \mathrm{U} / \mathrm{kg}(n=3), 1000 \mathrm{U} / \mathrm{kg}(n=3), 1600 \mathrm{U} / \mathrm{kg}(n=3)$ and $2500 \mathrm{U} / \mathrm{kg}(n=6)$. The median age was 57 years $(33-74) ; 87 \%$ were hepatitis B carriers and $47 \%$ had prior systemic treatment. The most commonly reported drug-related non-

T. Yau

Department of Medicine,

The University of Hong Kong, Queen Mary Hospital,

Pokfulam, Hong Kong, China

T. Yau $\cdot$ W. Chan $\cdot$ R. Pang $\cdot$ S. T. Fan $\cdot$ R. T. Poon $(\bowtie)$

Department of Surgery,

The University of Hong Kong, Queen Mary Hospital,

102 Pokfulam Road,

Pokfulam, Hong Kong, China

e-mail: poontp@hkucc.hku.hk

T. Yau $\cdot$ R. Pang $\cdot$ S. T. Fan $\cdot$ R. T. Poon

Centre for Cancer Research,

The University of Hong Kong, Queen Mary Hospital,

Pokfulam, Hong Kong, China

\section{S. T. Fan $\cdot$ R. T. Poon}

State Key Laboratory of Liver Diseases,

The University of Hong Kong, Queen Mary Hospital,

Pokfulam, Hong Kong, China haematological adverse events (AEs) were diarrhea (13.3\%), abdominal discomfort (6.7\%) and nausea (6.7\%). No drugrelated haematological AEs were seen. Only 1 of the six patients that received $2500 \mathrm{U} / \mathrm{kg}$ peg-rhArg1 experienced DLT (grade 4 bilirubin elevation) and thus the maximum tolerated dose was $2500 \mathrm{U} / \mathrm{kg}$. PK and PD analysis indicated that peg-rhArg1 was efficacious in inducing arginine depletion in a dose-dependent manner. Adequate arginine depletion dose was achieved in the 1,600$2,500 \mathrm{U} / \mathrm{kg}$ range and therefore the optimal biological dose was at $1600 \mathrm{U} / \mathrm{kg}$, which was chosen as the recommended dose. The best response was stable disease for $>8$ weeks in $26.7 \%$ of the enrolled patients. Conclusion Peg-rhArg 1 has manageable safety profile and preliminary evidence of activity in advanced HCC patients.

\section{P. Chan}

Department of Medicine, Ruttonjee Hospital,

266 Queen's Road East,

Wan Chai, Hong Kong

P. N. Cheng $\cdot$ L. Chen

Bio-Cancer Treatment International Limited,

Rm 511-513, 5/F, Bio-Informatics Centre,

2 Science Park West Avenue, Hong Kong Science Park,

Shatin, NT, Hong Kong

J. Yuen

Hong Kong Sanatorium \& Hospital,

2 Village Road,

Happy Valley, Hong Kong 
Keywords Arginine - Advanced HCC · Arginase · Peg-rhArg1

\section{Background}

Hepatocellular carcinoma (HCC) is the third most common cause of cancer-related death [1]. While long-term survival of patients with early HCC amenable to surgical treatment has improved in recent years, prognosis of patients with advanced disease remains poor with the median overall survival (OS) usually around 3 months from the time of diagnosis of the advanced disease [2].

HCC is relatively chemo-resistant. Thus far, there are no convincing phase III data to suggest that systemic chemotherapy, whether singly or in combination, improves overall survival in patients with advanced disease [3]. Notably, the use of the new generation of chemotherapy, such as oxaliplatin has recently demonstrated seemingly encouraging results. In particular, the results of the randomised phase 3 trial in comparing the efficacy of oxaliplatin plus 5Flurourcail and Leucovorin versus doxorubicin (FOLFOX4) as the palliative treatment for advanced HCC was reported in the ASCO meeting 2010 by Quinn et al [4]. The preliminary results showed that FOLFOX resulted in better overall survival and better tolerability than patients received doxorubicin. Thus, the future role of oxaliplatin in the treatment algorithm of advanced HCC patients is being investigated in the ongoing trials in advanced HCC population. Recently, two pivotal phase-III randomized placebo-controlled trials in the West [5] and Asia-Pacific region [6] have shown benefit with single agent sorafenib in patients with advanced HCC. Based on these studies, sorafenib was approved for the management of advanced HCC patients. It is noteworthy that the OS of patients on sorafenib was only 6.5 months, compared with 4.2 months in patients on placebo [6]. Thus, the overall improvement in OS is around 2.3 months. Furthermore, the treatment side effects from sorafenib, such as hand-foot-skin reaction and fatigue should not be lightly dismissed. Therefore, an unmet medical need remains to develop more effective therapeutic agents with significantly less side effects in the treatment of advanced HCC.

Arginine is a semi-essential amino acid involved in the synthesis of a wide range of peptides, proteins, and a myriad of metabolic pathways and cellular events [7]. HCC cells are said to be auxotrophic for arginine since they cannot, like normal somatic cells, recycle arginine from citrulline, making them completely reliant on exogenous arginine for growth. Thus, there are potential benefits in using arginine deprivation therapy in treating HCC. In vitro, arginine depletion strongly induced tumour regression in argininosuccinate synthase (ASS) deficient HCC cell lines [8]. In vivo, xenograft studies confirmed that arginine depletion inhibited tumour growth in murine $\mathrm{HCC}$, which do not express ASS mRNA. However, when it was transfected with an expression plasmid containing ASS cDNA, the transfected cells were much more resistant to arginine depletion $[8,9]$.

Two major enzyme therapeutics, pegylated arginine deiminase and pegylated human hepatic arginase, are now in various stages of clinical development. Arginine deiminase (ADI) is a Mycoplasma secreted enzyme that degrades arginine to citrulline in vivo, releasing ammonia as the other reaction product [10]. It may control growth of ASS deficient or arginine auxotrophic HCC. The pegylated form of ADI is now in phase-II clinical trial in HCC and melanoma and is reported to have moderate disease-stabilizing activity in HCC [11]. Arginase is another arginine-degrading enzyme for use in the treatment of arginine auxotrophic tumours. Unlike ADI, this non-xenogenic and native (human) enzyme hydrolyses arginine to ornithine and urea; the latter is non-toxic and excreted in the urine. The arginase used in this trial was of Good Manufacturing Practice (GMP) grade- pegylated recombinant human arginase1 (peg-rhArg1), from an E. coli expression system. After suitable pegylation to increase its in vivo half-life in plasma from a few hours to 3-4 days, it has been shown to have strong in vitro and in vivo anti-tumour activities in a number of tumour types including HCC [9]. Production of this pegylated recombinant human arginase 1 has been reported elsewhere [12].

The aim of this open-label, phase I dose-escalation study was to evaluate safety, pharmacokinetics (PK)/pharmacodynamics (PD), and potential anti-tumor activity of pegrhArg1 in patients with advanced HCC.

\section{Patients and methods}

This was a single-center, prospective, open-label, phase 1 dose-escalation study of peg-rhArg1 in subjects with advanced HCC unsuitable for either surgical or loco-regional therapy. The protocol was approved by the local IRB and Ethics Committee with written consents being obtained from the patients before enrollment. The trial was registered in clinicaltrials.gov identifier NCT01092091 and conducted in compliance with ICH guidance of Good Clinical Practice.

\section{Patient's eligibility}

Advanced HCC patients not suitable for surgery or various loco-regional therapies at the Queen Mary Hospital, Hong Kong, were recruited. HCC was diagnosed either by cytohistological confirmation or by non-invasive criteria according to the European Association for Study of Liver disease (EASL) criteria: cirrhotic patients with either two coincident imaging techniques demonstrating focal lesion $>2 \mathrm{~cm}$ with 
Table 1 Patient cohorts and dosing scheme

\begin{tabular}{lr}
\hline Cohort & $\mathrm{U} / \mathrm{kg}$ \\
\hline One & 500 \\
Two & 1000 \\
Three & 1600 \\
Four & 2500 \\
\hline
\end{tabular}

arterial hypervascularization or one imaging technique showing focal lesion $>2 \mathrm{~cm}$ with arterial hypervascularization and associated with alpha fetal protein (AFP) level $>400 \mathrm{ng} / \mathrm{ml}$ [13]. Staging was by American Joint Committee on Cancer (AJCC) and Barcelona Clinic Liver Cancer (BCLC) score. The eligibility criteria included adult patients 18-75 years old; patients with Child-Pugh class A or B cirrhosis; Karnofsky performance status (KPS) $\geq 80 \%$; expected life expectancy of $\geq 12$ weeks and with adequate organ function: complete blood picture (absolute neutrophil count $(\mathrm{ANC})>1.0 \times 10^{9} / \mathrm{L}$, platelet count $>100 \times 10^{9} / \mathrm{L}$ ) and biochemistry (total bilirubin of $\leq 2 \times$ upper limit of normal, serum AST and ALT $\leq 5 \times$ upper limit of normal). The disease must be measurable with at least one lesion, which was at least $2 \mathrm{~cm}$ in one dimension either on CT or MRI scan. All the enrolled patients had clear progressive disease with their last treatment modalities prior to study entry.

Dosing scheme, dose limiting toxicity and maximum tolerated dose

Patient enrollment was based on the $3+3$ paradigm with doseescalating cohorts as shown in Table 1. The escalation scheme followed the modified Fibonacci scheme commonly used in phase I trials [14]. DLT was defined as any grade 4 toxicities, grade 3 neutropenia or the occurrence of neutropenic sepsis, grade 3 thrombocytopenia or any grade 3 toxicities that did not return to grade $1 / 2$ within 3 weeks, except for alopecia. The maximum tolerated dose (MTD) was defined as the highest weekly level of IV bolus peg-rhArg1 at which no more than one of six patients experienced a DLT.

Initially, a cohort of 3 advanced HCC patients received a bolus I.V. administration of peg-rhAgr1 started at $500 \mathrm{U} / \mathrm{kg}$. Single dose safety parameters, including hematology and chemistry laboratory profiles, were monitored weekly for 2 weeks. Patients did not demonstrate a dose limiting toxicity ( DLT) following the single dose subsequently received weekly IV bolus of peg-rhArg1 at the same dose level from week 3 (day 15) onwards (Fig. 1).

Pharmacokinetics(PK) and Pharmacodynamics (PD) measurement

Plasma arginase and corresponding arginine levels were measured following bolus I.V. administration of pegrhArg1 in groups of 3 patients given a range of pegrhArgI. After the dose for each patient, blood samples for plasma arginase were drawn at baseline, 2 and $4 \mathrm{~h}$ after the single dosing to establish maximum plasma concentration $\left(\mathrm{C}_{\max }\right)$ and initial clearance. Additional single time-point samples were drawn on Days 2, 4, 6 and 8 to establish the terminal $T_{1 / 2}$ and duration of arginine depletion after the first dose. During weekly dosing, baseline (pre-dose) samples and $24 \mathrm{~h}$ post dose samples were obtained from weeks 3 to 5 ; then only one baseline sample from weeks 6 to 11 . PK parameters to be calculated based on a non-compartmental model approach include: $\mathrm{C}_{\max }$, time to maximum observed concentration $\left(\mathrm{T}_{\max }\right)$, minimum observed concentration $\left(\mathrm{C}_{\text {min }}\right)$, and area under the curve $\left(\mathrm{AUC}_{\infty}\right)$. Arginase levels were assessed using ELISA Kit (BEIJING 4A BIOTECH Co. LTD, in China). The methodology of these tests is per standard ELISA protocol. Arginine level was analyzed by a high-speed amino acid analyzer [15]. Adequate Arginine Depletion (ADD) dose was defined as the dose of pegrhAgr1 that depletes arginine level to $<8 \mu \mathrm{M}$ throughout
Fig. 1 Dose escalating scheme of Peg-rhArg1

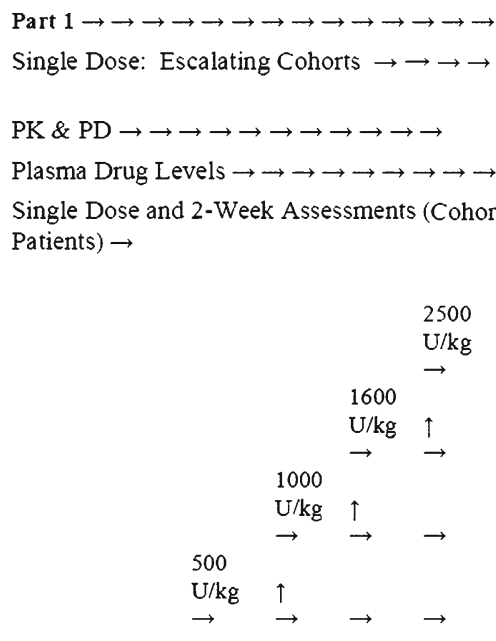

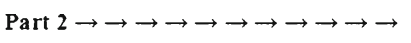

Multiple Dose $\rightarrow \rightarrow \rightarrow \rightarrow \rightarrow \rightarrow \rightarrow \rightarrow$

PK \& PD $\rightarrow \rightarrow \rightarrow \rightarrow \rightarrow \rightarrow \rightarrow$

Arginine Depletion $\rightarrow \rightarrow \rightarrow \rightarrow \rightarrow \rightarrow \rightarrow$ Weekly Monotherapy Dose $\rightarrow \rightarrow \rightarrow \rightarrow \rightarrow$ 
the treatment period. Optimal Biological Dose (OBD), which was defined as the lowest dose of peg-rhArg1 to achieve sustained ADD.

\section{Objectives}

The primary objectives of this study were the safety, tolerability and in establishing the safety dose of peg-rhArg1 in the future clinical trials of advanced HCC patients. Secondary objectives included characterization of PK/PD and evaluation of treatment efficacy per RECIST 1.0 guideline [16].

Disease evaluation

A full history and complete physical examination, including performance status were performed at every clinical visit. Disease assessment was performed by CT scan at baseline, week 11 and week 21 . Response was independently reviewed by a designated radiologist at the Hong Kong Sanatorium and Hospital throughout the study and classified according to RECIST 1.0 criteria [16]. Patients who completed more than 8 weeks of the study were considered as evaluable for response. Weekly peg-rhArg1 administration continued (without dose escalation) for patients whose disease assessments showed either stable disease (SD) or response.

\section{Safety monitoring}

Safety assessments consisted of monitoring and recording all the adverse and serious adverse events (AEs). Apart from monitoring of vital signs, regular collection of urine, hematology and blood chemistry of the enrolled subjects were performed. Patients who received at least one weekly dose of peg-rhArg1 were defined as Intent-to-treat population and considered evaluable for safety. AEs were evaluated according to National Cancer Institute Criteria (NCIC) version 3.0 [17].

\section{Statistical analysis}

Survival analysis was computed by the Kaplan-Meier method. Progression-free survival (PFS) was calculated from the date of commencement of study drugs to the date of documented progression or death. OS was calculated from the date of commencement of study drugs to the date of death or last follow-up. The analysis was performed on intent-to-treat basis. All statistical analysis was performed by Statistical Package for Social Science version 13.0 for Windows (SPSS Inc., Chicago, IL USA).

\section{Results}

Demographics

Between May 2008 and July 2009, 15 patients were recruited in the study at weekly doses of $500 \mathrm{U} / \mathrm{kg}$ (3 patients), $1000 \mathrm{U} / \mathrm{kg}$ (3 patients), $1600 \mathrm{U} / \mathrm{kg}$ (3 patients) and $2500 \mathrm{U} / \mathrm{kg}$ (6 patients). Table 2 shows the demographics of all the recruited subjects. The median age was 57 years, and 12 patients $(80 \%)$ were male. All except 2 were hepatitis B carriers; 2 received antiviral treatment for hepatitis B during peg-rhAgr1 treatment. Eleven (73.3\%) patients were in Child-Pugh A cirrhosis, while the remaining four (26.7\%) were in Child-Pugh B cirrhosis. All the recruited patients had advanced HCC that belonged to BCLC stage C (100\%). Table 3 shows the details of the prior treatments in the enrolled patients. Ten $(67 \%)$ patients had received prior treatments for $\mathrm{HCC}$, while the remaining 5 patients were treatment naïve. Notably, seven (47\%) patients were heavily pretreated with various chemotherapy regimens and antiVEGF therapies.

\section{Safety and tolerability}

The median treatment exposure was 10 weeks (range, 2-23 weeks). Half of the patients had 6 to 12 weeks treatment exposure. Seven patients did not complete the 8 weeks of study drug. One out of three patients experienced possible DLT, in cohort $4(2500 \mathrm{U} / \mathrm{kg})$ with grade 4 bilirubin elevation in the first month of treatment. An additional 3 patients were recruited into this cohort as per protocol requirement. No further DLT was reported in these additional 3 patients. Therefore, the MTD was defined as $2500 \mathrm{U} / \mathrm{kg}$.

In total, 9 possible drug-related AEs were recorded (Table 4). Most peg-rhArg1-related toxicities were grade 1 or 2, which resolved after discontinuation of peg-rhArg1. No treatment-related deaths were reported.

\section{Pharmacokinetics}

Figure 2a shows the pharmacokinetics of peg-rhArg1 in cohort 1-3. Following the first dose of peg-rhArg1, the mean $\mathrm{C}_{\max }$ at $500,1000,1600 \mathrm{U} / \mathrm{kg}$ were $78.8(86.5 \%)$, $212.0(35.2 \%)$, and $171.3(17.3 \%) \mu \mathrm{g} / \mathrm{ml}$, respectively. The mean peak-through ratios were 4.23 in cohort 2 , and ranged from 2 to 3 for other 2 cohorts. The mean $\mathrm{AUC}_{\infty}$ was about $8.94(28.2 \%) \mathrm{mg} * \mathrm{~h} / \mathrm{ml}$ for cohort 1 $(500 \mathrm{U} / \mathrm{kg})$. Cohort $2(1000 \mathrm{U} / \mathrm{kg})$ and $3(1600 \mathrm{U} / \mathrm{kg}) \mathrm{had}$ similar mean $\mathrm{AUC}_{\infty}$, which were 34.67 (58.4\%) and $33.36(20.5 \%) \mathrm{mg} * \mathrm{~h} / \mathrm{ml}$ respectively. 
Table 2 Demographics, baseline characteristic and prior treatment of 15 patients administrated with Peg-rhArg1

\begin{tabular}{|c|c|c|}
\hline & $\begin{array}{l}\text { No. of } \\
\text { Patients }\end{array}$ & $\begin{array}{l}\text { Percentage } \\
(\%)\end{array}$ \\
\hline \multicolumn{3}{|l|}{ Characteristics } \\
\hline \multicolumn{3}{|l|}{ Sex } \\
\hline Male & 12 & 80 \\
\hline Female & 3 & 20 \\
\hline \multicolumn{3}{|l|}{ Age (years) } \\
\hline Median & 57 & \\
\hline Range & $33-74$ & \\
\hline \multicolumn{3}{|c|}{ Karnofsky Performance Scale } \\
\hline 100 & 6 & 40 \\
\hline 90 & 7 & 46.7 \\
\hline 80 & 2 & 13.3 \\
\hline \multicolumn{3}{|l|}{ Hepatitis Serology } \\
\hline Hep Bs Ag positive & 13 & 86.7 \\
\hline \multicolumn{3}{|l|}{ Child-Pugh Status } \\
\hline A & 11 & 73.3 \\
\hline $\mathrm{B}$ & 4 & 26.7 \\
\hline \multicolumn{3}{|c|}{ Alpha-fetal Protein (AFP) } \\
\hline$<=400$ & 5 & 33.3 \\
\hline$>400$ & 10 & 66.7 \\
\hline \multicolumn{3}{|c|}{$\begin{array}{l}\text { Disease stage at the time of study } \\
\text { entry } \\
\text { AJCC Staging }\end{array}$} \\
\hline IIIA & 4 & 26.7 \\
\hline IIIC & 3 & 20 \\
\hline IV & 8 & 53.3 \\
\hline \multicolumn{3}{|l|}{ BCLC Staging } \\
\hline $\mathrm{C}$ & 15 & 100 \\
\hline \multicolumn{3}{|l|}{ Distant Metastases } \\
\hline Lung & 6 & 40 \\
\hline Bone & 1 & 6.7 \\
\hline Adrenal & 2 & 13.3 \\
\hline Brain & 1 & 6.7 \\
\hline \multicolumn{3}{|c|}{ Invasion of Major Vessels } \\
\hline Portal vein invasion & 5 & 33.3 \\
\hline \multicolumn{3}{|l|}{ Prior Treatment } \\
\hline \multicolumn{3}{|l|}{ Surgical treatment } \\
\hline Liver resection & 5 & 33.3 \\
\hline Laparotomy & 1 & 6.7 \\
\hline \multicolumn{3}{|c|}{ Local ablative procedures } \\
\hline TACE & 6 & 40 \\
\hline RFA & 1 & 6.7 \\
\hline Systemic therapy & 7 & 46.7 \\
\hline
\end{tabular}

AJCC American Joint Committee on Cancer staging; BCLC Barcelona Clinic Liver Cancer staging; Hep Bs Ag Hepatitis B surface antigen; TACE Transarterial chemo-embolization; RFA Radiofrequency ablation
Table 3 Summary of prior treatments in enrolled subjects

\begin{tabular}{|c|c|}
\hline Subject Number & Prior Treatment Summary \\
\hline 101 & Right Hepatectomy \\
\hline 102 & TACE (Cisplatin) : $3 \mathrm{X}$ \\
\hline 201 & $\begin{array}{l}\text { Liver Resection; } \\
\text { Radiofrequency Ablation; } \\
\text { Doxorubicin: } 6 \text { cycles; } \\
\text { TACE (Cisplatin): } 2 \mathrm{X} \text {; } \\
\text { Anti-VEGF: } 6 \text { cycles }\end{array}$ \\
\hline 202 & $\begin{array}{l}\text { Right Hepatectomy; } \\
\text { Doxorubicin: } 6 \text { cycles; } \\
\text { Anti-VEGF: } 6 \text { cycles }\end{array}$ \\
\hline 203 & $\begin{array}{l}\text { Sorafenib: } 8 \text { cycles; } \\
\text { Capecitabine: } 8 \text { cycles; } \\
\text { Oxaliplatin: } 8 \text { cycles; } \\
\text { TACE (Cisplatin): } 4 X\end{array}$ \\
\hline 301 & $\begin{array}{l}\text { TACE (Cisplatin): } 4 \mathrm{X} \\
\text { Anti-VEGF: } 9 \text { cycles }\end{array}$ \\
\hline 302 & Anti-VEGF: 4 cycles \\
\hline 403 & $\begin{array}{l}\text { Hepatectomy; } \\
\text { Anti-VEGF: } 2 \text { cycles } \\
\text { TACE (Cisplatin): } 8 \mathrm{X}\end{array}$ \\
\hline 405 & $\begin{array}{l}\text { Doxorubicin: } 3 \text { cycles; } \\
\text { Anti-VEGF: } 14 \text { cycles; } \\
\text { TACE (Cisplatin) : } 8 \mathrm{X}\end{array}$ \\
\hline 406 & Hepatectomy only \\
\hline
\end{tabular}

TACE Transarterial chemo-embolization; Anti-VEGF Anti vascular endothelial growth factor

\section{Pharmacodynamics}

Peg-rhArg1 was efficacious in inducing arginine depletion in a dose-dependent manner. Figure $2 b$ shows the relationship between plasma arginine levels after peg-rhArg1 dosing. The time taken to achieve undetectable plasma of arginine was $2 \mathrm{~h}$ for all patients following a single dose. The $2500 \mathrm{U} / \mathrm{kg}$ dose level in cohort 4 had the greatest arginine depletion and the highest median duration of the ADD effect (166 h). In cohort 3 (1600U/kg), the effective ADD was achieved after the second weekly dose of peg-rhArg1 and ADD was maintained throughout the treatment period. The lowest dose of pegrhAgrl that achieved ADD was $1600 \mathrm{U} / \mathrm{kg}$ in cohort 3 and this dose was therefore deemed the OBD.

\section{Recommended safe dose of Peg-rhArg1}

The study was terminated on completion of all the 6 cases in cohort 4 since both the MTD (2500 U/kg) and OBD $(1600 \mathrm{U} / \mathrm{kg})$ were established. The final recommended safe dose for future clinical trials was chosen as $1600 \mathrm{U} / \mathrm{kg}$ instead of $2500 \mathrm{U} / \mathrm{kg}$ based on the premise that it was the 
Table 4 Summary of possible treatment related Adverse Events (AEs)

Total No. of AEs

\begin{tabular}{llll}
\hline $\begin{array}{l}\text { Grade 1 } \\
(\%)\end{array}$ & $\begin{array}{l}\text { Grade 2 } \\
(\%)\end{array}$ & $\begin{array}{l}\text { Grade 3 } \\
(\%)\end{array}$ & $\begin{array}{l}\text { Grade 4 } \\
(\%)\end{array}$ \\
\hline
\end{tabular}

\begin{tabular}{lllll} 
Abdomen Pain & $1(6.7)$ & & & \\
Diarrhea & $2(13.3)$ & & & \\
Nausea & & $1(6.7)$ & & \\
Elevated ALT & & & $1(6.7)$ & \\
Elevated AST & & $1(6.7)$ & \\
Elevated & & $1(6.7)$ & $1(6.7)$ \\
$\quad$ Bilirubin & & $1(6.7)$ & \\
Elevated GGT & & & \\
\hline
\end{tabular}

All AEs were scored according to the National Cancer institute (NCI) Common Terminology Criteria, Version 3, June 10, 2003

ALT Alanine Transaminase; AST Asparate Transaminase; GGT Gamma-glutamyl transferase

lower tolerated dose of peg-rhArg1 to achieve adequate arginine depletion.

Treatment efficacy and survival analysis

Of the 15 patients recruited, 7 patients were terminated before formal radiological assessments for efficacy due to the following reasons: 2 died of liver failure from advanced cirrhosis, 3 patients died of rapid progressive diseases, 1 had prolonged elevation of bilirubin (DLT) and 1 withdrew voluntarily from treatment. Overall, 4 (26.7\%) patients achieved SD and was observed at all 4 dose levels, one from each cohort. Notably, marked tumor necrosis was observed in two cases without actual change in tumor dimensions on CT (Fig. 3). The median baseline alphafetal protein (AFP) level was $954 \mu \mathrm{g} / \mathrm{L}$ (range, 4-267000). Among all the 15 enrolled patients, six patients (40\%) showed a decline in AFP compared with baseline during the study.

The median PFS and OS for the entire study population of 15 patients were 2.8 months (95\% CI: $0.2-3.0$ months) (Fig. 4) and 5.1 months (95\% CI: $0.2-14.8$ months) respectively. The patients with Child-Pugh A cirrhosis had significantly better median OS than the Child-Pugh B cirrhotic patients ( 8.5 versus 1.8 months, $p<0.008)$. Two patients receiving peg-rhArg1 at $1000 \mathrm{U} / \mathrm{kg}$ and $1600 \mathrm{U} / \mathrm{kg}$ remained SD for $>6$ months.

\section{Conclusion}

This is the first human clinical study using peg-rhArg1 in the treatment of advanced human malignancy. We have demonstrated that arginine depletion in humans can be achieved safely with peg-rhAgr1 in a dose-response manner. There was no treatment-related death and all the AEs, except for the one possible case of DLT in cohort 4 , were mild grade 1 and 2 non-hematologic toxicities, such as nausea and abdominal discomfort, which resolved spontaneously with symptomatic treatment. Thus, the primary objectives of the study were met and the recommended weekly dose for future development of Peg-rhAgr1 was chosen as $1600 \mathrm{U} / \mathrm{kg}$.

As in any drug trials involving patients with severely compromised liver functions, there is always a problem in attributing the exact cause of dysfunction, whether due to the test drug or progression of underlying liver disease, i.e. cirrhosis, hepatitis or malignancy, often all three. Overall, only 2 of the 15 patients in the entire study had possible severe drug-related liver dysfunction, one in cohort 1 and the other in cohort 4 . The first patient had grade 3 elevation of ALT/AST after the first injection and was taken off the study. Before he went into hepatic encephalopathy with liver failure, a CT scan confirmed progression of the liver tumour, from which he died after 2 months off treatment. The second case had grade 3 followed by 4 hyperbilirubinemia after a single injection of peg-rhArg1. Since the patient had no overt clinical signs of disease progression, this hyperbilibrubinemia was deemed as DLT. Although great care was taken to recruit patients with adequate life-expectancy into the study, the poor outcomes of these patients early on in their treatment illustrate how unpredictable the clinical course of heavily pre-treated HCC patients with advanced disease can be. In particular, nearly half of the recruited patients were heavily pre-treated with various prior system therapies, which might account for the rapid deterioration of the recruited patients in the study. As per protocol requirement, the additional 3 cases were recruited into cohort 4 , and it was reassuring that no further liver DLT was observed. The study was terminated on completion of all the 6 cases in cohort 4 since the MTD and OBD - the two important objective parameters the authors aimed to establish - had already been found.

In terms of toxicity profiles, peg-rhAgr1 does have an advantage over both ADI and sorafenib. When compared with ADI, peg-rhAgr1 releases urea as a side product, which is much less toxic than the ammonia that is released by ADI treatment. This is important since in patients with compromised liver function, as in most HCC cases, the extra ammonia may make the patients more susceptible to develop HE. Indeed, the most common AEs attributed to ADI include transient and reversible HE. Other toxicities reported in ADI included skin and injection site necrosis and electrolyte disturbance, which were not seen in our study. Being a Mycoplasma product, $\mathrm{ADI}$ is a xeonbiotic immunogenic substance, despite pegylation. It has been reported that neutralizing antibodies to ADI developed $\sim 50$ days after initiation of treatment [11]. Peg- 

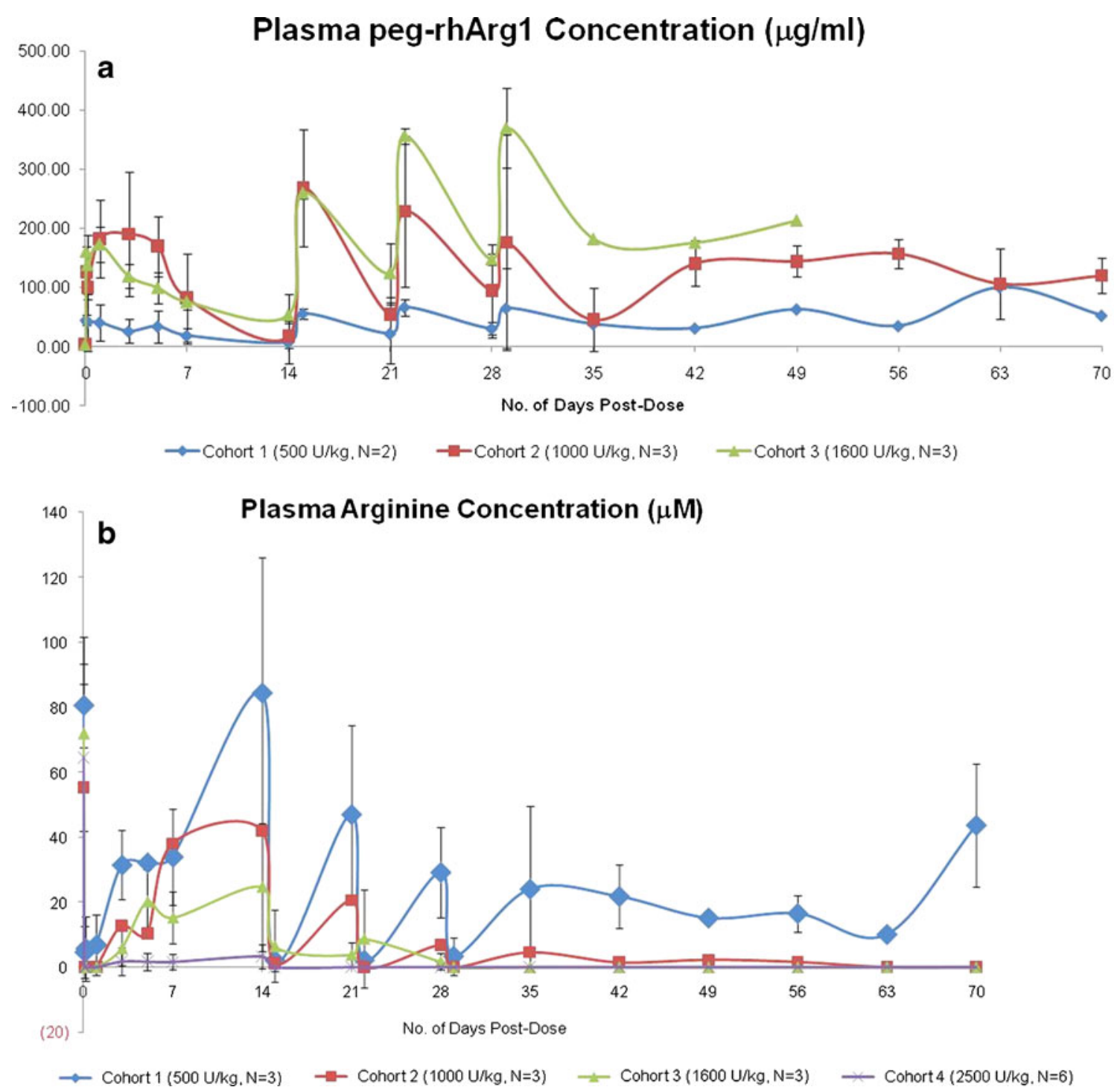

Fig. 2 a. Plasma Peg-rhArg1 level after single and multiple doses of Peg-rhArg1 in HCC patients b. Plasma arginine concentration after single and multiple dosing of Peg-rhArg1 in HCC patients

rhAgr1, on the other hand, is a recombinant human hepatic enzyme and in our preclinical and clinical studies (data not shown); no evidence of immunogenicity has been found to date. This has important therapeutic implication since the

Fig. 3 Computerized Tomographic (CT) Scan of HCC Patient: Serial CT scan images of liver tumor before treatment, 11 weeks after treatment showing the increase of necrosis with diameter of $25.6 \mathrm{~mm}$, and 27 weeks after treatment showing necrosis with diameter of $71.8 \mathrm{~mm}$, respectively

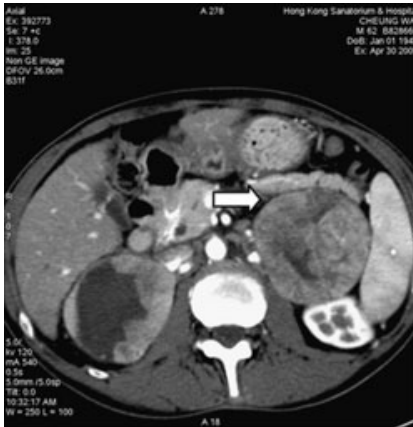

Apr 2009

Before treatment

neutralizing antibodies would render the drug ineffective as an arginine-depleting agent. It was also reported in the ADI studies that arginine levels would rise in the second month of treatment, coinciding with the development of neutralizing

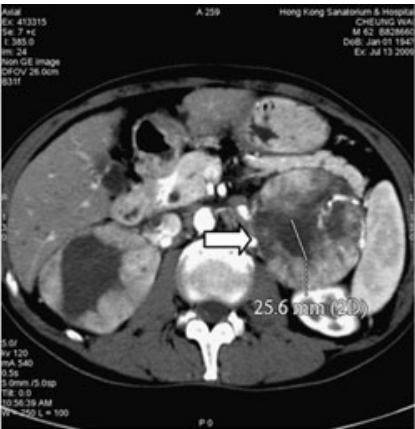

July 2009

11 week after treatment

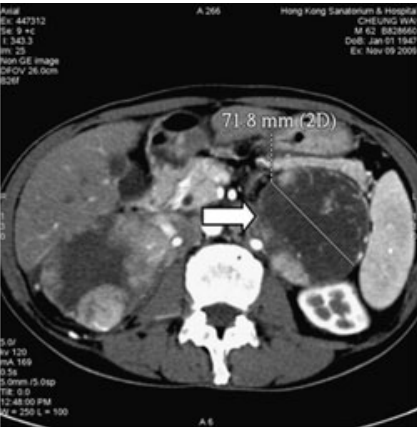

Nov 2009

27 week after treatment 
Fig. 4 Kaplan-Meier

Progression-free Survival (PFS)

of enrolled patients
Progression Free Survival for Arginase treated Advance HCC patient

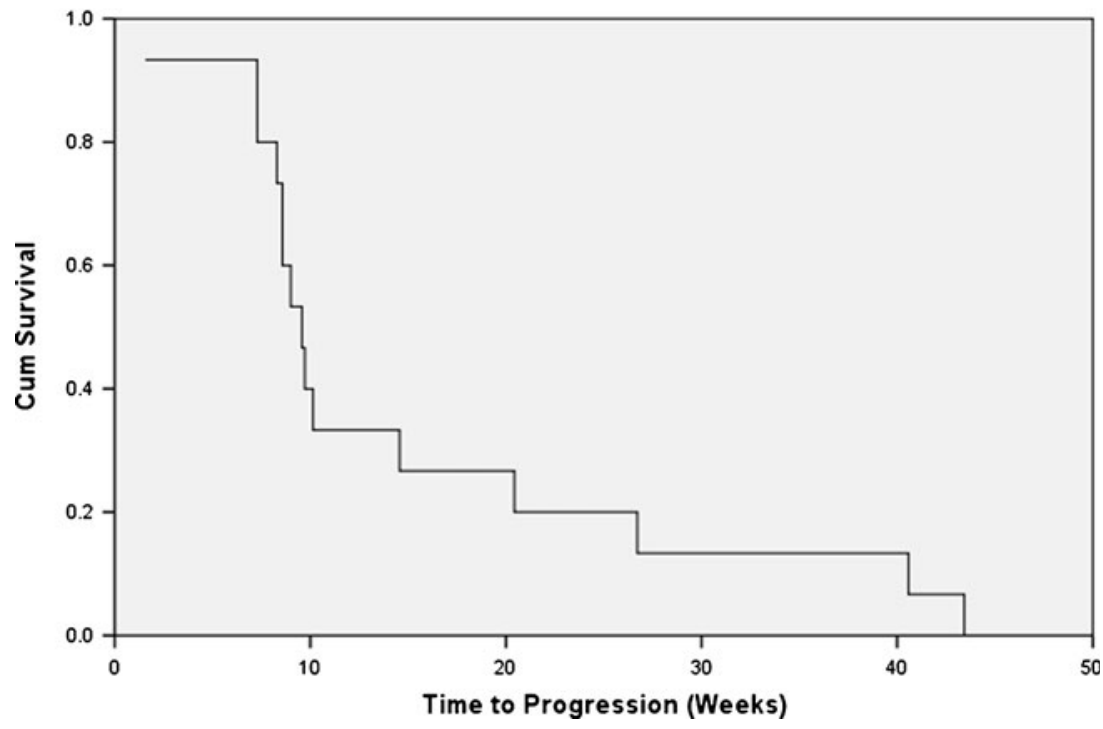

antibodies to ADI. On the other hand, neutralizing antibodies to Peg-rhAgr1 have not been detected thus far in the present study and it is reassuring that arginine levels remained at lower levels throughout the entire study as long as treatment continued. More importantly, the AEs profile of peg-rhAgr1 also compares favourably with that of sorafenib, which has large numbers of grade III and IV toxicities in terms of hand-footskin reaction, hypertension and rash. Again, none of these were seen in this study.

Enzymatic depletion or deprivation therapy, whether with peg-rhAgr1 or ADI, has now emerged as a new platform of anti-cancer treatment in a number of human malignancies, such as HCC and melanoma [18]. In vitro and in vivo xenograft studies suggest that this mode of treatment is efficacious also in prostatic and renal cell cancers, in which ASS and arginosuccinate lyase (ASL) were highly repressed or absent in an MD Anderson series [19]. Pegylated human arginase has recently been shown in one US study to induce remission in acute T cell leukemia [20]. The present study serves to prove the concept that arginine depletion is really a promising approach in treating advanced HCC patients. Thus, further evaluation of peg-rhArg1 in future phase-II trials of advanced HCC is warranted.

Peg-rhAgr1 might have demonstrated early activity in the group of heavily pre-treated advanced HCC patients. Of the 8 cases completed 8 weeks of treatment and beyond, 4 had durable SD lasting for $3 \mathrm{~m}+, 6 \mathrm{~m}+, 6 \mathrm{~m}+$ and $3 \mathrm{~m}+$, respectively with drop in AFP level. One patient had minor shrinkage of the target lung lesions that did not qualify as a partial response by the RECIST 1.0 criteria. As experienced by other clinical investigators in the field of HCC, assessing CT response to treatment in HCC is notoriously difficult and tumour response may result in tumour necrosis instead of shrinkage (Fig. 2) [21]. Notably, at the time when this study was conducted, RECIST 1.0 criteria was the standard for assessing tumor response [22]. As yet, there is no consensus in the HCC community about which new radiological criteria, such as EASL [13] or modified RECIST [21] would be a better method to assess response in $\mathrm{HCC}$ patients receiving targeted therapy or biological therapy alone. Unfortunately, 7 enrolled patients were not evaluable for treatment response and most of these patients discontinued the therapy early mainly due to either rapid disease progression or various complications from underlying cirrhosis. This indeed reflects the challenges in conducting clinical trials in this refractory patient population with aggressive tumour biology and poor liver reserve.

In conclusion, peg-rhArg1 has a manageable safety profile and is potentially a superior arginine depleting agent than ADI in the treatment of human malignancies due to its low toxicity profile and sustainable arginine depletion. The optimal therapeutic dose of peg-rhArgI is $1600 \mathrm{U} / \mathrm{kg}$ in humans for future studies in advanced HCC patients.

Conflicts of Interests Dr Thomas Yau, Pierre Chan, William Chan, Jimmy Yuen, Roberta Pang, Professor ST Fan and Professor Ronnie Poon report no potential conflict of interest.

Dr PN Cheng is the chief executive officer of Bio-Cancer Treatment International Limited. Dr Li Chen is the chief technical officer of BioCancer Treatment International Limited

Financial Support Bio-Cancer Treatment International Limited and the University of Hong Kong Hepatocellular Carcinoma Research Grant

Open Access This article is distributed under the terms of the Creative Commons Attribution License which permits any use, distribution, and reproduction in any medium, provided the original author(s) and the source are credited. 


\section{References}

1. Parkin DM (2001) Global cancer statistics in the year 2000. Lancet Oncol 2:533-543

2. Yau T, Yao TJ, Chan P et al (2008) A new prognostic score system in patients with advanced hepatocellular carcinoma not amendable to locoregional therapy: implication for patient selection in systemic therapy trials. Cancer 113:2742-2751

3. Palmer DH, Hussain SA, Johnson PJ (2004) Systemic therapies for hepatocellular carcinoma. Expert Opin Investig Drugs 13:15551568

4. Qin S, Bai Y, Ye S et al (2010) Phase III study of oxaliplatin plus 5fluorouracil/leucovorin (FOLFOX4) versus doxorubicin as palliative systemic chemotherapy in advanced HCC in Asian patients. American Society of Clinical Oncology Annual Meeting Chicago. J Clin Oncol 28:15, (suppl; abstr 4008)

5. Llovet JM, Ricci S, Mazzaferro V et al (2008) Sorafenib in advanced hepatocellular carcinoma. N Engl J Med 359:378-390

6. Cheng AL, Kang YK, Chen Z et al (2009) Efficacy and safety of sorafenib in patients in the Asia-Pacific region with advanced hepatocellular carcinoma: a phase III randomised, double-blind, placebo-controlled trial. Lancet Oncol 10:25-34

7. Morris SM Jr (2002) Regulation of enzymes of the urea cycle and arginine metabolism. Annu Rev Nutr 22:87-105

8. Ensor CM, Holtsberg FW, Bomalaski JS, Clark MA (2002) Pegylated arginine deiminase (ADI-SS PEG20,000 mw) inhibits human melanomas and hepatocellular carcinomas in vitro and in vivo. Cancer Res 62:5443-5450

9. Cheng PN, Lam TL, Lam WM et al (2007) Pegylated recombinant human arginase (rhArg-peg5,000mw) inhibits the in vitro and in vivo proliferation of human hepatocellular carcinoma through arginine depletion. Cancer Res 67:309-317

10. Ni Y, Schwaneberg U, Sun ZH (2008) Arginine deiminase, a potential anti-tumor drug. Cancer Lett 261:1-11

11. Glazer ES, Piccirillo M, Albino V et al (2010) Phase II study of pegylated arginine deiminase for nonresectable and metastatic hepatocellular carcinoma. J Clin Oncol 28:2220-2226

12. Tsui SM, Lam WM, Lam TL et al (2009) Pegylated derivatives of recombinant human arginase (rhArg1) for sustained in vivo activity in cancer therapy: preparation, characterization and analysis of their pharmacodynamics in vivo and in vitro and action upon hepatocellular carcinoma cell (HCC). Cancer Cell Int 9:9

13. Bruix J, Sherman M, Llovet JM et al (2001) Clinical management of hepatocellular carcinoma. Conclusions of the Barcelona-2000 EASL conference. European Association for the Study of the Liver. J Hepatol 35:421-430

14. Le Tourneau C, Lee JJ, Siu LL (2009) Dose escalation methods in phase I cancer clinical trials. J Natl Cancer Inst 101:708-720

15. Cheng PN, Leung YC, Lo WH, Tsui SM, Lam KC (2005) Remission of hepatocellular carcinoma with arginine depletion induced by systemic release of endogenous hepatic arginase due to transhepatic arterial embolisation, augmented by high-dose insulin: arginase as a potential drug candidate for hepatocellular carcinoma. Cancer Lett 224:67-80

16. Therasse P, Arbuck SG, Eisenhauer EA et al (2000) New guidelines to evaluate the response to treatment in solid tumors. European Organization for Research and Treatment of Cancer, National Cancer Institute of the United States, National Cancer Institute of Canada. J Natl Cancer Inst 92:205-216

17. Trotti A, Colevas AD, Setser A et al (2003) CTCAE v3.0: development of a comprehensive grading system for the adverse effects of cancer treatment. Semin Radiat Oncol 13:176-181

18. Ascierto PA, Scala S, Castello G et al (2005) Pegylated arginine deiminase treatment of patients with metastatic melanoma: results from phase I and II studies. J Clin Oncol 23:7660-7668

19. Dillon BJ, Prieto VG, Curley SA et al (2004) Incidence and distribution of argininosuccinate synthetase deficiency in human cancers: a method for identifying cancers sensitive to arginine deprivation. Cancer 100:826-833

20. Hernandez CP, Morrow K, Lopez-Barcons LA et al (2010) Pegylated arginase I: a potential therapeutic approach in T-ALL. Blood 115:5214-5221

21. Llovet JM, Di Bisceglie AM, Bruix J et al (2008) Design and endpoints of clinical trials in hepatocellular carcinoma. J Natl Cancer Inst 100:698-711

22. Therasse P, Arbuck SG, Eisenhauer EA et al (2000) New guidelines to evaluate the response to treatment in solid tumors. J Natl Cancer Inst 92:205-216 